MCTP-05-91

\title{
A Minimal Spontaneously Broken Hidden Sector and its Impact on Higgs Boson Physics at the Large Hadron Collider
}

\author{
Robert Schabinger and James D. Wells \\ Michigan Center for Theoretical Physics (MCTP) \\ University of Michigan, Ann Arbor, MI 48109-1120, USA
}

\begin{abstract}
Little experimental data bears on the question of whether there is a spontaneously broken hidden sector that has no Standard Model quantum numbers. Here we discuss the prospects of finding evidence for such a hidden sector through renormalizable interactions of the Standard Model Higgs boson with a Higgs boson of the hidden sector. We find that the lightest Higgs boson in this scenario has smaller rates in standard detection channels, and it can have a sizeable invisible final state branching fraction. Details of the hidden sector determine whether the overall width of the lightest state is smaller or larger than the Standard Model width. We compute observable rates, total widths and invisible decay branching fractions within the general framework. We also introduce the "A-Higgs Model", which corresponds to the limit of a hidden sector Higgs boson weakly mixing with the Standard Model Higgs boson. This model has only one free parameter in addition to the mass of the light Higgs state and it illustrates most of the generic phenomenology issues, thereby enabling it to be a good benchmark theory for collider searches. We end by presenting an analogous supersymmetry model with similar phenomenology, which involves hidden sector Higgs bosons interacting with MSSM Higgs bosons through D-terms.
\end{abstract}

September 2005 
There are many deficiencies of the Standard Model (SM), such as the hierarchy problem, flavor problem, dark matter problem, cosmological constant problem, electroweak symmetry breaking problem, CP violation problem, baryogenesis problem, etc. Much of the effort in particle theory has been to posit solutions to these problems and ask how they would show up at experiments. The presence of a hidden sector, defined here to mean extra states that have no SM gauge charge but are charged under some other exotic gauge symmetry, does not necessarily solve any of the problems above. However, there are many extensions of the SM that purport to solve these problems that do have hidden sectors as part of their generic constructions, as is well-known to be the case for supersymmetry and string theory.

In this paper we take no position on what the primary motivation for a hidden sector might be, but rather consider the most simple hidden sector possible and ask what effect it would have on LHC phenomenology [1]. Our beginning assumptions are that there exists a hidden sector Higgs boson $\Phi$ which can decay into undetectable hidden sector states with total width $\Gamma^{\text {hid }}$, and that the SM Higgs state interacts only with SM particles in the standard way. Our analysis will be for a condensing $\Phi$ fundamental scalar. This is in contrast to other analyses which assume a singlet scalar state that has no vacuum expectation value. The advantage of that latter possibility is that the hidden sector singlet state can be stable and could be the cold dark matter. This interesting possibility has been studied well in the literature 2]. We, however, study the case of $\langle\Phi\rangle \neq 0$ since we like to think of the hidden sector as having a rich gauge theory structure which is at least partly broken by $\langle\Phi\rangle \neq 0$. It is possible here that the lightest hidden sector particle could be the dark matter, but we only require for this analysis that the hidden sector states do not decay into SM particles within the detector.

We comment at the start that a full precision electroweak analysis is not possible in this study, since we are looking at generic aspects of only Higgs physics and so are in effect summing over all the possibilities for other hidden sector states such as $Z^{\prime}$ couplings and mass that would affect a detailed fit. There is much freedom to have additional Higgs bosons and $Z^{\prime}$ bosons conspire to yield a reasonable fit to the precision electroweak data [3], and so we suggest that our approach is not any less applicable by not fully considering electroweak precision data.

For our study, we will assume that the hidden sector has at least one gauge symmetry $G_{h i d}$ that is broken by a vacuum expectation value of the Higgs boson $\Phi$. There are two opportunities for a renormalizable coupling between states of the hidden sector and those of 
the SM. The first possibility for mixing between states at the renormalizable level is kinetic mixing among the gauge bosons of $U(1)_{Y}$ and a $U(1)_{h i d}$. Recall that for abelian gauge symmetry the field-strength tensor $B_{\mu \nu}=\partial_{\mu} B_{\nu}-\partial_{\nu} B_{\mu}$ is gauge invariant, and thus an interaction operator is allowed between the field-strengths of two different $U(1)$ symmetries,

$$
\mathcal{L}_{m i x}=\chi B_{\mu \nu} C^{\mu \nu}
$$

where $\chi$ is some dimensionless mixing parameter. The phenomenology for theories with this kind of interaction is interesting [4] however, we will not focus on that here, partly because we do not want to confine ourselves to discussions that have applicability only to hidden sectors with abelian symmetries, and partly because the precision electroweak fit sensitivity to this operator is higher than the one we discuss below and being constrained as such would be less likely to lead to profound impacts at the LHC.

Instead, we focus on the experimental implications of the renormalizable interaction of the SM Higgs boson with the hidden sector Higgs boson $|H|^{2}|\Phi|^{2}[5]$, which is a 4-dimensional operator and gauge invariant. The Higgs boson lagrangian under consideration for this case is

$$
\mathcal{L}_{\text {Higgs }}=\left|D_{\mu} H\right|^{2}+\left|D_{\mu} \Phi\right|^{2}+m_{H}^{2}|H|^{2}+m_{\Phi}^{2}|\Phi|^{2}-\lambda|H|^{4}-\rho|\Phi|^{4}+\eta|H|^{2}|\Phi|^{2}
$$

Generically, for a stable potential that admits vevs for $H$ and $\Phi$ the parameters $m_{H}^{2}, m_{\Phi}^{2}, \lambda$ and $\rho$ are all positive. On the other hand, $\eta$ is not generically required to be of one particular sign. For simplicity, we are assuming that $\Phi$ is a Higgs boson that breaks a $U(1)_{\text {hid }}$ symmetry; however, the results that follow easily generalize to $\Phi$ being a Higgs boson that breaks any hidden sector group spontaneously.

The component fields can be written as

$$
H=\frac{1}{\sqrt{2}}\left(\begin{array}{c}
h+v+i G^{0} \\
G^{ \pm}
\end{array}\right), \quad \Phi=\frac{1}{\sqrt{2}}\left(\phi+\xi+i G^{\prime}\right)
$$

where $v(\simeq 246 \mathrm{GeV})$ and $\xi$ are vacuum expectation values about which the $H$ and $\Phi$ fields are expanded. The $G$ fields are Goldstone bosons absorbed by the vector bosons, and so no physical pseudo-scalar states are left in the spectrum. However, the scalar spectrum has two physical states rather than just the one of the SM. In terms of the $\{h, \phi\}$ interaction eigenstates, the mass matrix one must diagonalized to obtain the two physical mass eigenstates is

$$
\mathcal{M}^{2}=\left(\begin{array}{cc}
2 \lambda v^{2} & \eta v \xi \\
\eta v \xi & 2 \rho \xi^{2}
\end{array}\right)
$$


This matrix is diagonalized by the mixing angle

$$
\tan \omega=\frac{\eta v \xi}{\left(\rho \xi^{2}-\lambda v^{2}\right)+\sqrt{\left(\rho \xi^{2}-\lambda v^{2}\right)^{2}+\eta^{2} v^{2} \xi^{2}}}
$$

with

$$
\begin{aligned}
h & =\cos \omega s_{1}+\sin \omega s_{2} \\
\phi & =-\sin \omega s_{1}+\cos \omega s_{2}
\end{aligned}
$$

The masses of the two eigenstates are then

$$
m_{s_{1}, s_{2}}^{2}=\left(\lambda v^{2}+\rho \xi^{2}\right) \pm \sqrt{\left(\lambda v^{2}-\rho \xi^{2}\right)^{2}+\eta^{2} v^{2} \xi^{2}}
$$

When $\eta \rightarrow 0$ we recover the two diagonal eigenstates $m_{s_{1}}^{2}=2 \lambda v^{2}$ and $m_{s_{2}}^{2}=2 \rho \xi^{2}$.

Within the SM, the couplings of the Higgs boson to SM particles is completely determined by the Higgs boson mass. This in turn leads to a completely specified partial width of SM Higgs decay into any of its kinematically allowed final states. We denote these partial widths as $\Gamma_{i}^{\mathrm{SM}}\left(m_{h}\right)$. The total width is $\Gamma^{\mathrm{SM}}\left(m_{h}\right)$ with no index. The equivalent total width of the hidden sector Higgs boson is $\Gamma^{\text {hid }}\left(m_{\phi}\right)$. In other words, $\Gamma^{\mathrm{SM}}$ and $\Gamma^{\text {hid }}$ are the widths of the SM Higgs boson and the hidden sector boson, respectively, in the case of no mixing. We assume that all final states of $\Gamma^{\text {hid }}$ are invisible to particle detectors.

When $h$ and $\phi$ mix to form eigenstates $s_{1}$ and $s_{2}$ the decays will in general be to both SM states and hidden sector states. For the sake of simplicity in writing subsequent formula, we assume that $m_{s_{1}}<m_{s_{2}}$ and we write $c_{\omega} \equiv \cos \omega$ and $s_{\omega} \equiv \sin \omega$. If $m_{s_{2}}>2 m_{s_{1}}$ the decay $s_{2} \rightarrow s_{1}+s_{1}$ would be allowed kinematically. The partial width of this decay depends on the parameters of the mixing lagrangian of eq. 2. If we isolate the $\Delta \mathcal{L}_{\text {mix }}=\mu s_{1}^{2} s_{2}$ contribution in the lagrangian, where $\mu$ has dimensions of mass and is determined by the vevs of the $H$ and $\Phi$ fields as well as their dimensionless interaction coefficients, we find

$$
\mu=\frac{\eta}{2}\left(\xi c_{\omega}^{3}+v s_{\omega}^{3}\right)+(3 \lambda-\eta) v c_{\omega}^{2} s_{\omega}+(3 \rho-\eta) \xi c_{\omega} s_{\omega}^{2}
$$

(Note, when the $\eta$ dependence of $s_{\omega}$ is taken into account, $\mu \rightarrow 0$ when $\eta \rightarrow 0$, as it should.) The resulting partial width of $s_{2} \rightarrow s_{1} s_{1}$ is

$$
\Gamma\left(s_{2} \rightarrow s_{1} s_{1}\right)=\frac{|\mu|^{2}}{4 \pi m_{s_{2}}} \sqrt{1-\frac{4 m_{s_{1}}^{2}}{m_{s_{2}}^{2}}}
$$

These Higgs-to-Higgs decays are tell-tale signs of Higgs mixing operator(s). 
Production of $s_{1}$ and $s_{2}$ is entirely determined by the strength of their interactions with respect to the SM. In the case of $V V \rightarrow h$, where $V V=g g, W W, Z Z$, we know that to first approximation it is proportional to the Higgs boson partial width

$$
\sigma(V V \rightarrow h)\left(m_{h}\right) \propto \Gamma(h \rightarrow V V)\left(m_{h}\right)
$$

Therefore, the production cross-sections of $s_{1}$ is related to that of the SM simply by $c_{\omega}^{2}$

$$
\sigma\left(V V \rightarrow s_{1}\right)\left(m_{s_{1}}\right)=c_{\omega}^{2} \sigma(V V \rightarrow h)\left(m_{s_{1}}\right)
$$

For $s_{2}$ the ratio is $s_{\omega}^{2}$. One should take note that this implies that there is no state in the spectrum that has a production cross-section as large as the SM Higgs boson.

The branching fractions of the lighter state $s_{1}$ into a SM final state $i$ is

$$
B_{i}\left(s_{1}\right)=\frac{c_{\omega}^{2} \Gamma_{i}^{\mathrm{SM}}\left(m_{s_{1}}\right)}{c_{\omega}^{2} \Gamma^{\mathrm{SM}}\left(m_{s_{1}}\right)+s_{\omega}^{2} \Gamma^{\mathrm{hid}}\left(m_{s_{1}}\right)}
$$

If $\Gamma^{\text {hid }}\left(m_{s_{1}}\right) \simeq 0$, which would be the case if there were no hidden sector states light enough for the mixed $s_{1}$ boson to decay into, the branching fraction would be the same as that of the SM. The overall width, on the other hand, would be smaller by a factor of $c_{\omega}^{2}$. This case is equivalent to the "universal suppression of Higgs boson observables" discussed in ref. [6]. In this case, light and narrow width Higgs bosons $\left(115 \mathrm{GeV} \lesssim m_{s_{1}} \lesssim 160 \mathrm{GeV}\right)$ become extraordinarily difficult to find at colliders. For a heavier $s_{1}$ boson, the smaller cross-section associated with $c_{\omega}^{2}$ suppression will be compensated for somewhat by the more narrow width. Discerning $s_{1} \rightarrow Z Z \rightarrow 4 l$ above background is of course aided by a smaller width if the width is well above the detector's invariant mass resolution.

If $\Gamma^{\text {hid }}\left(m_{s_{1}}\right) \neq 0$, the lightest Higgs state may decay predominantly into undetectable particles depending on how large $\Gamma^{\text {hid }}$ and $s_{\omega}$ are. The invisible branching fraction is

$$
B_{\mathrm{inv}}\left(s_{1}\right)=\frac{s_{\omega}^{2} \Gamma^{\mathrm{hid}}\left(m_{s_{1}}\right)}{c_{\omega}^{2} \Gamma^{\mathrm{SM}}\left(m_{s_{1}}\right)+s_{\omega}^{2} \Gamma^{\mathrm{hid}}\left(m_{s_{1}}\right)}
$$

which approaches 1 when $s_{\omega}^{2} \Gamma^{\text {hid }} \gg c_{\omega}^{2} \Gamma^{\mathrm{SM}}$. In this case, one has the double problem of suppressed production plus invisible decays. For the most difficult case of the light Higgs boson, the SM decay width is already quite small by accident (i.e., when $m_{b} \ll m_{s_{1}}<2 m_{W}$ ), and so it would not take much for $\Gamma^{\text {hid }}$ to dominate over $\Gamma^{\mathrm{SM}}$, resulting in an invisibly decaying Higgs boson. The detectability of this kind of state at a hadron collider is quite challenging even without the production suppression, although we expect that a high-energy $e^{+} e^{-}$collider would have relatively little trouble with the invisible decay aspect [7]. 
All of the above discussion for $s_{1}$ applies to the case of $s_{2}$ except the mixing factors change in an obvious way, and there is the new possibility of $s_{2} \rightarrow s_{1} s_{1}$ decays. Thus, the production cross-section is suppressed compared to the SM by

$$
\sigma\left(V V \rightarrow s_{2}\right)=s_{\omega}^{2} \sigma(V V \rightarrow h)
$$

and the branching fractions into SM final states are

$$
B_{i}\left(m_{s_{2}}\right)=\frac{s_{\omega}^{2} \Gamma_{i}^{\mathrm{SM}}\left(m_{s_{2}}\right)}{s_{\omega}^{2} \Gamma_{i}^{\mathrm{SM}}\left(m_{s_{2}}\right)+c_{\omega}^{2} \Gamma_{i}^{\mathrm{SM}}\left(m_{s_{2}}\right)+\Gamma\left(s_{2} \rightarrow s_{1} s_{1}\right)}
$$

Similarly, the branching fractions to invisible final states and $s_{1} s_{1}$ final states, which subsequently decay into a myriad of possibilities, are determined by

$$
\begin{aligned}
& B_{\mathrm{inv}}\left(s_{2}\right)=\frac{c_{\omega}^{2} \Gamma^{\mathrm{hid}}\left(m_{s_{2}}\right)}{c_{\omega}^{2} \Gamma^{\mathrm{SM}}\left(m_{s_{2}}\right)+s_{\omega}^{2} \Gamma^{\mathrm{hid}}\left(m_{s_{2}}\right)+\Gamma\left(s_{2} \rightarrow s_{1} s_{1}\right)} \text { and } \\
& B\left(s_{2} \rightarrow s_{1} s_{1}\right)=\frac{\Gamma\left(s_{2} \rightarrow s_{1} s_{1}\right)}{c_{\omega}^{2} \Gamma^{\mathrm{SM}}\left(m_{s_{2}}\right)+s_{\omega}^{2} \Gamma^{\text {hid }}\left(m_{s_{2}}\right)+\Gamma\left(s_{2} \rightarrow s_{1} s_{1}\right)}
\end{aligned}
$$

Let us focus on the detectability of the lightest Higgs mass eigenstate $s_{1}$. The phenomenology of this case is determined by three input parameters:

$$
m_{s_{1}}, s_{\omega}^{2}, \text { and } r_{s_{1}} \equiv \frac{\Gamma^{\mathrm{hid}}\left(m_{s_{1}}\right)}{\Gamma^{\mathrm{SM}}\left(m_{s_{1}}\right)}
$$

In terms of these input parameters, the total rate of Higgs-mediated events at a hadron collider, such as $g g \rightarrow h \rightarrow \gamma \gamma, Z Z, W W, t \bar{t}, \ldots$ is related to the SM rate by

$$
\frac{\sigma_{i} B_{j}}{\sigma_{i}^{\mathrm{SM}} B_{j}^{\mathrm{SM}}}=\frac{\left(1-s_{\omega}^{2}\right)^{2}}{1-\left(1-r_{s_{1}}\right) s_{\omega}^{2}}
$$

The index $i$ refers to the initial state that created the Higgs boson, and $j$ refers to the states into which the Higgs boson decays. The total width of the $s_{1}$ Higgs boson, which determines the broadness of the reconstructed invariant mass peak, is related to the SM width by

$$
\frac{\Gamma\left(m_{s_{1}}\right)}{\Gamma^{\mathrm{SM}}\left(m_{s_{1}}\right)}=1-\left(1-r_{s_{1}}\right) s_{\omega}^{2}
$$

Finally, the branching fraction of the $s_{1}$ into hidden sector (invisible) final states is

$$
B_{\text {inv }}\left(m_{s_{1}}\right)=\frac{s_{\omega}^{2} r_{s_{1}}}{1-\left(1-r_{s_{1}}\right) s_{\omega}^{2}}
$$




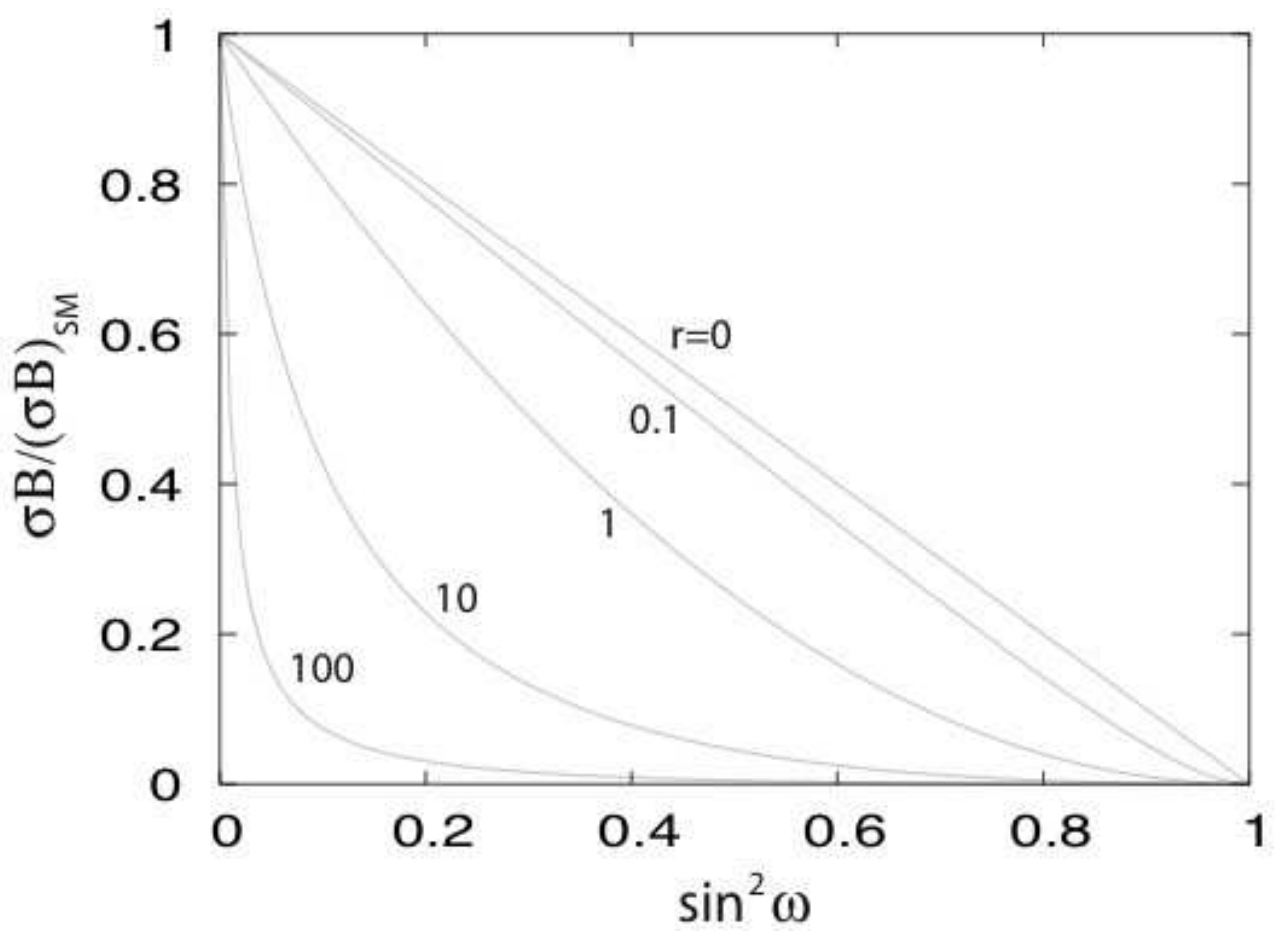

Figure 1: The rate of Higgs boson observables $\sigma_{i} B_{j}$ relative to that of the $\mathrm{SM}$ for various values of $r=\Gamma^{\text {hid }} / \Gamma^{\mathrm{SM}}=0,0.1,1,10,100$ (from top to bottom). Note, the rate never exceeds that of the SM, thus making detection of hidden sector mixed Higgs bosons more challenging than detection of the SM Higgs boson.

In Figs. 11, 2] and 3] we plot for various values of $r_{s_{1}}$ the three observables $\sigma_{i} B_{j} /\left(\sigma_{i} B_{j}\right)_{\mathrm{SM}}$, $\Gamma / \Gamma^{\mathrm{SM}}$ and $B_{\mathrm{inv}}$. In Fig. 1 we see that the rate for Higgs boson induced observables never exceeds that of the SM. This obviously makes detection of the $s_{1}$ Higgs boson in the standard channels much more difficult than detection of the SM Higgs boson.

In Fig. 2 we see that the total width can be quite a bit larger than the SM Higgs boson. Combining this fact with the results of Fig. 1, which showed that the rate is always suppressed, implies that discovering the $s_{1}$ in the standard detection channels at the LHC may take significantly more luminosity and care than is required for the SM Higgs boson. On the other hand, if $r<1$, an interesting experimental question comes into play. In that case, as $s_{\omega}^{2}$ increases, the observable rate into standard channels goes down (bad for detection) whereas the width decreases (good for detection). It is a technical experimental question as to whether there is any mass range for $s_{1}$ where the narrowing of the peak helps more than the dropping of the rate hurts. We suppose that if that ever is the case it would be in the high mass region, well above the invariant mass resolution of the detector. 


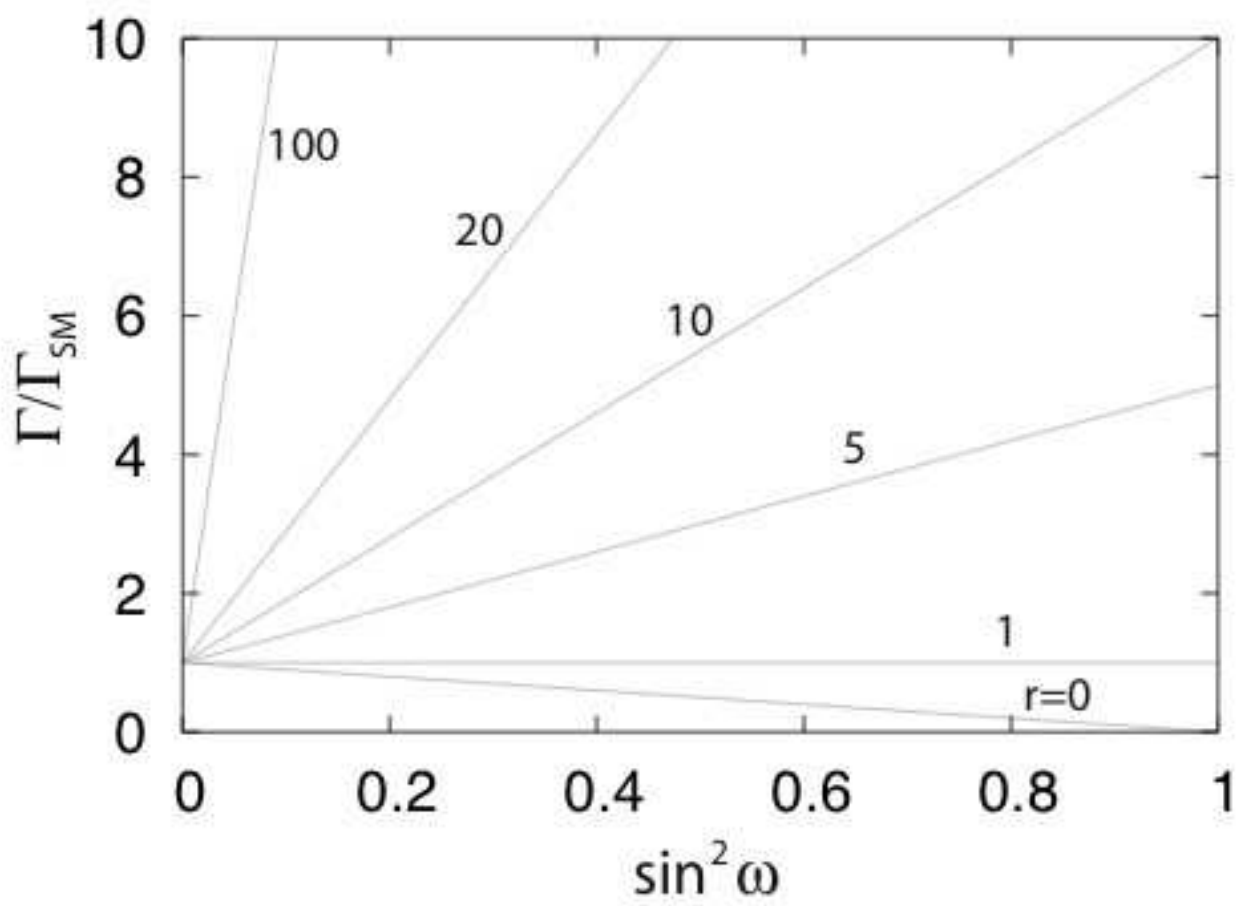

Figure 2: The total width compared to that of the SM total width for various values of $r=\Gamma^{\text {hid }} / \Gamma^{\mathrm{SM}}=0,1,5,10,20,100$ (from bottom to top).

In Fig. 3 we plot the invisible width of the $s_{1}$ Higgs boson. For large values of $s_{\omega}^{2}$ the hope of detecting the $s_{1}$ Higgs boson in an invisible channel is quite small, since the total rate is small and invisible final states are notoriously difficult at hadron colliders [8, 9, 10]. Even for rather small values of $s_{\omega}^{2}$ the invisible Higgs rate could be the most important signal for the light $s_{1}$ boson if $\Gamma^{\text {hid }} \gg \Gamma^{\mathrm{SM}}$. In that case, the $s_{2}$ boson would also like to decay invisibly, making detection of any Higgs boson of the theory quite challenging.

There is an interesting limit of this framework to analyze that is approximated by $\Gamma^{\text {hid }} \gg$ $\Gamma^{\mathrm{SM}}$ (i.e., $r \rightarrow \infty$ ) and $s_{\omega}^{2} \rightarrow 0$, but $r s_{\omega}^{2} \rightarrow A$. In this case,

$$
\frac{\sigma_{i}}{\sigma_{i}^{\mathrm{SM}}}=1, \quad \frac{\sigma_{i} B_{j}}{\sigma_{i}^{\mathrm{SM}} B_{j}^{\mathrm{SM}}}=\frac{1}{1+A}, \quad \frac{\Gamma\left(m_{s_{1}}\right)}{\Gamma^{\mathrm{SM}}\left(m_{s_{1}}\right)}=1+A, \quad \text { and } \quad B_{\mathrm{inv}}\left(m_{s_{1}}\right)=\frac{A}{1+A}
$$

The $s_{2}$ state is not produced in this limit. Although the production cross-section for $s_{1}$ is the same as the SM Higgs boson, the total rate into standard detectability channels, such as $\gamma \gamma$ or $Z Z$, falls as $A$ increases. At the same time the total width increases, making these standard channels even more challenging. On the other hand, as $A$ increases, the invisible branching fraction increases, and the techniques for discovering invisibly decaying Higgs bosons become important [8, 9, 10]. 


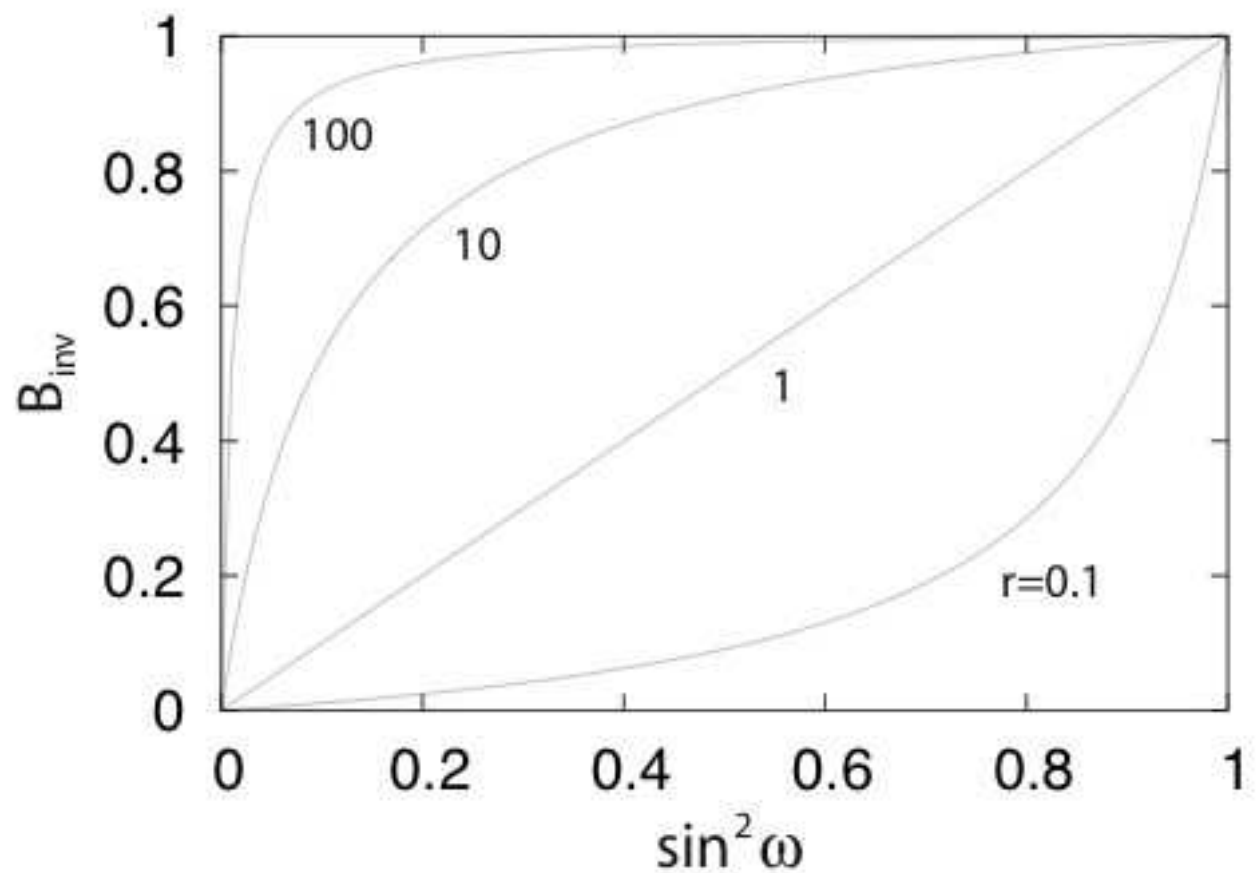

Figure 3: The invisible branching fraction of the lightest Higgs boson $s_{1}$ for various values of $r=\Gamma^{\text {hid }} / \Gamma^{\mathrm{SM}}=0.1,1,10,100$ (from bottom to top). The contour of $r=0$ corresponds to the $B_{\text {inv }}=0$ line along the $x$-axis.

We consider this model to be interesting physically, as it implies that there is very little interaction between our sector and the hidden sector but the hidden sector has large decay width into its own hidden sector states compared to the SM Higgs boson. This is especially interesting for a rather light Higgs boson $(\lesssim 160 \mathrm{GeV})$ which as we discussed above accidentally has a small width into SM states. Thus, the "A-Higgs model" of eq. 223 would be interesting to study in detail in our view since it is motivated physically, has only two free parameters $\left(m_{s_{1}}\right.$ and $A$ ) and illustrates much of the generic phenomenology relevant to hidden sector Higgs mixing (reduced rates, increased widths and invisible decays).

Finally, we comment on the supersymmetric analogue of this case. By the definition of hidden sector we have given above, a prime supersymmetry candidate would be the $S$ field in $\Delta W=\lambda S H_{u} H_{d}$. If $S$ is charged under another $U(1)$ then its vacuum expectation value would break that symmetry, which fits into our general discussion well. An added bonus is that the vev would generate the $\mu$ term through $\lambda\langle S\rangle=\mu$. This scenario has been studied quite extensively in the literature [11], and we do not have anything additional to say regarding its LHC phenomenology. 
A slightly "more hidden" sector in supersymmetry, which is closer to the spirit of the SM hidden sector discussed above, is a field $\Phi$ that is charged under a new $U(1)_{\text {hid }}$ and participates in a $D$-term interaction with the SM Higgs bosons, but has no gauge-invariant, renormalizable interaction in the superpotential. Of course, a $D$-term interaction would require the $H_{u}$ and $H_{d}$ fields to be charged under $U(1)_{\text {hid }}$ as well. Although $\Phi H_{u} H_{d}$ is not allowed in the superpotential by assumption here, the $D$ term interaction between the states yields a mixing in close analogy with our SM case above:

$$
V=\frac{g_{\text {hid }}^{2}}{2}\left(Q_{u}\left|H_{u}\right|^{2}+Q_{d}\left|H_{d}\right|^{2}+|\Phi|^{2}+\cdots\right)^{2}
$$

For simplicity we are normalizing the $U(1)_{\text {hid }}$ such that $\Phi$ has charge +1 . Assuming the MSSM soft lagrangian for $H_{u}$ and $H_{d}$, and adding a soft mass for $\Phi$ such that $\langle\Phi\rangle \neq 0$, we can construct the CP-even tree-level Higgs mass matrix in the $\left\{h_{u}, h_{d}, \phi\right\}$ basis, where $\operatorname{Re}\left(H_{u}\right)=\left(h_{u}+v\right) / \sqrt{2}, \operatorname{Re}\left(H_{d}\right)=\left(h_{d}+v_{d}\right) / \sqrt{2}$ and $\operatorname{Re}(\Phi)=(\phi+\xi) / \sqrt{2}$ :

$\mathcal{M}^{2}=\left(\begin{array}{ccc}m_{A}^{2} c_{\beta}^{2}+\left(m_{Z}^{2}+m_{Z^{\prime}}^{2} \gamma^{2} Q_{u}^{2}\right) s_{\beta}^{2} & -\left(m_{A}^{2}+m_{Z}^{2}-m_{Z^{\prime}}^{2} \gamma^{2} Q_{u} Q_{d}\right) s_{\beta} c_{\beta} & m_{Z^{\prime}}^{2} \gamma Q_{u} s_{\beta} \\ -\left(m_{A}^{2}+m_{Z}^{2}-m_{Z^{\prime}}^{2} \gamma^{2} Q_{u} Q_{d}\right) s_{\beta} c_{\beta} & m_{A}^{2} s_{\beta}^{2}+\left(m_{Z}^{2}+m_{Z^{\prime}}^{2} \gamma^{2} Q_{d}^{2}\right) c_{\beta}^{2} & m_{Z^{\prime}}^{2} \gamma Q_{d} c_{\beta} \\ m_{Z^{\prime}}^{2} \gamma Q_{u} s_{\beta} & m_{Z^{\prime}}^{2} \gamma Q_{d} c_{\beta} & m_{Z^{\prime}}^{2}\end{array}\right)$

In terms of the gauge couplings and vevs, $m_{Z^{\prime}}^{2}=g_{\text {hid }}^{2} \xi^{2}$ and $\gamma^{2}=\left(v_{u}^{2}+v_{d}^{2}\right) / \xi^{2}$. The values of $c_{\beta}$ and $s_{\beta}$ are obtained from $t_{\beta}=\tan \beta=v_{u} / v_{d}$. This matrix is now $3 \times 3$ in contrast to the $2 \times 2$ matrix of the MSSM due to the additional Higgs field.

The upper bound of the lightest Higgs boson state of this supersymmetric theory can be obtained by finding the lightest eigenvalue of the upper two-by-two matrix, which is

$$
m_{s_{1}}^{2} \leq m_{Z}^{2} c_{\beta}^{2}+m_{Z^{\prime}}^{2} \gamma^{2}\left(Q_{d} c_{\beta}^{2}+Q_{u} s_{\beta}^{2}\right)^{2}+\Delta_{\mathrm{rad}}
$$

where $\Delta_{\text {rad }}$ is the quantum correction whose leading contribution comes from the top-stop loops of the MSSM. For heavy $m_{A}$ and heavy $m_{Z^{\prime}}$ the bound given above becomes the actual eigenvalue. In the MSSM, $\Delta_{\text {rad }}$ generally needs to be above $\sim(70 \mathrm{GeV})^{2}$ in order for $m_{h}$ to be above the experimental limit of $114 \mathrm{GeV}$. Such a high radiative correction is not easy to obtain within weak-scale supersymmetry, and thus strains our ordinary view of naturalness [12]. The lightest Higgs mass in the MSSM has a maximum mass of about $130 \mathrm{GeV}$ for TeV-scale supersymmmetry. Furthermore, much of the supersymmetry parameter space that is considered "natural", which is associated with lighter weak-scale superpartner masses, leads to a prediction of the mass lighter than the current experimental limit of $114 \mathrm{GeV}$. Thus, the extra $m_{Z^{\prime}}^{2} \gamma^{2}$ contribution to the mass eigenvalue is welcome. 
Nevertheless, we still expect the lightest Higgs mass to stay relatively light even in this theory

since $m_{Z^{\prime}}^{2} \gamma^{2} \sim m_{Z}^{2}$. Therefore, the Higgs boson width into SM states remains narrow (again, $m_{b} \ll m_{s_{1}}<2 m_{W}$ ), and the Higgs boson detection phenomenology of this supersymmetric theory is very similar to that of the SM-variant that we discussed earlier. All equations that were derived above for the SM hidden sector case carry forward with little change except to take care of the extra mixing angles in a $3 \times 3$ matrix.

The simple illustrative case of large $\Gamma^{\text {hid }}$ and small mixing within this supersymmetric context yields a similar result to what we found in the non-supersymmetric case: the lightest Higgs boson will have large suppressions of standard detection final state rates at the LHC, and there will be a premium on good analyses that keep free the total rates, the widths of the Higgs bosons and the branching fraction into invisible final states. The "A-Higgs model" discussed earlier is the simplest illustration of all these effects.

\section{Acknowledgements}

This work was supported in part by the Department of Energy, the Michigan Center for Theoretical Physics, and the NSF REU program. We thank J. Kumar, S. Martin and M. Toharia for helpful discussions.

\section{References}

[1] For work on related Higgs phenomenology, see T. Binoth and J. J. van der Bij, Z. Phys. C 75, 17 (1997) hep-ph/9608245; R. Akhoury, J. J. van der Bij and H. Wang, Eur. Phys. J. C 20, 497 (2001) hep-ph/0010187; T. Han, P. Langacker and B. McElrath, Phys. Rev. D 70, 115006 (2004) hep-ph/0405244.

[2] J. McDonald, Phys. Rev. D 50, 3637 (1994). C. P. Burgess, M. Pospelov and T. ter Veldhuis, Nucl. Phys. B 619, 709 (2001) hep-ph/0011335. H. Davoudiasl, R. Kitano, T. Li and H. Murayama, Phys. Lett. B 609, 117 (2005) hep-ph/0405097.

[3] See, for example, M. E. Peskin and J. D. Wells, Phys. Rev. D 64, 093003 (2001) hep-ph/0101342.

[4] K. S. Babu, C. F. Kolda and J. March-Russell, Phys. Rev. D 57, 6788 (1998) hep-ph/9710441. 
[5] R. Schabinger, "The Phenomenological Possibilities Introduced by an Exotic Scalar," Honors Thesis, University of Michigan, Ann Arbor, April 2005.

[6] J. D. Wells, "Extra dimensions and the universal suppression of Higgs boson observables at high energy colliders," SUSY 2002 (DESY, Hamburg), hep-ph/0205328.

[7] O. J. P. Eboli, M. C. Gonzalez-Garcia, A. Lopez-Fernandez, S. F. Novaes and J. W. F. Valle, Nucl. Phys. B 421, 65 (1994) hep-ph/9312278.

[8] J. F. Gunion, Phys. Rev. Lett. 72, 199 (1994) hep-ph/9309216. D. Choudhury and D. P. Roy, Phys. Lett. B 322, 368 (1994) hep-ph/9312347. S. G. Frederiksen, N. Johnson, G. L. Kane and J. Reid, Phys. Rev. D 50 (1994) 4244. O. J. P. Eboli and D. Zeppenfeld, Phys. Lett. B 495, 147 (2000) hep-ph/0009158.

[9] A. Datta and A. Raychaudhuri, Phys. Rev. D 57, 2940 (1998) hep-ph/9708444. B. P. Kersevan, M. Malawski and E. Richter-Was, Eur. Phys. J. C 29, 541 (2003) hep-ph/0207014. R. M. Godbole, M. Guchait, K. Mazumdar, S. Moretti and D. P. Roy, Phys. Lett. B 571, 184 (2003) hep-ph/0304137. M. Battaglia, D. Dominici, J. F. Gunion and J. D. Wells, hep-ph/0402062, K. Belotsky, V. A. Khoze, A. D. Martin and M. G. Ryskin, Eur. Phys. J. C 36, 503 (2004) hep-ph/0406037. H. Davoudiasl, T. Han and H. E. Logan, Phys. Rev. D 71, 115007 (2005) hep-ph/0412269.

[10] S. P. Martin and J. D. Wells, Phys. Rev. D 60, 035006 (1999) hep-ph/9903259. B. A. Dobrescu, G. Landsberg and K. T. Matchev, Phys. Rev. D 63, 075003 (2001) hep-ph/0005308.

[11] For some recent discussions of collider probes of the NMSSM see U. Ellwanger, J. F. Gunion and C. Hugonie, hep-ph/0503203. G. Moortgat-Pick, S. Hesselbach, F. Franke and H. Fraas, hep-ph/0502036. D. J. Miller, R. Nevzorov and P. M. Zerwas, Nucl. Phys. B 681, 3 (2004) hep-ph/0304049. U. Ellwanger, J. F. Gunion, C. Hugonie and S. Moretti, hep-ph/0401228.

[12] See, for example, G. W. Anderson, D. J. Castano and A. Riotto, Phys. Rev. D 55, 2950 (1997) hep-ph/9609463. G. L. Kane, T. T. Wang, B. D. Nelson and L. T. Wang, Phys. Rev. D 71, 035006 (2005) hep-ph/0407001. 